\title{
Learning a nonmediated route for response selection in task switching
}

\author{
Darryl W. Schneider • Gordon D. Logan
}

Published online: 7 February 2015

(C) Psychonomic Society, Inc. 2015

\begin{abstract}
Two modes of response selection-a mediated route involving categorization and a nonmediated route involving instance-based memory retrieval-have been proposed to explain response congruency effects in taskswitching situations. In the present study, we sought a better understanding of the development and characteristics of the nonmediated route. In two experiments involving training and transfer phases, we investigated practice effects at the level of individual target presentations, transfer effects associated with changing category-response mappings, target-specific effects from comparisons of old and new targets during transfer, and the percentages of early responses associated with task-nonspecific response selection (the target preceded the task cue on every trial). The training results suggested that the nonmediated route is quickly learned in the context of target-cue order and becomes increasingly involved in response selection with practice. The transfer results suggested that the target-response instances underlying the nonmediated route involve abstract response labels coding response congruency that can be rapidly remapped to alternative responses, but not rewritten when category-response mappings change after practice. Implications for understanding the nonmediated route and its relationship with the mediated route are discussed.
\end{abstract}

D. W. Schneider

Purdue University, West Lafayette, IN, USA

D. W. Schneider • G. D. Logan

Vanderbilt University, Nashville, TN, USA

D. W. Schneider $(\square)$

Department of Psychological Sciences, Purdue University,

West Lafayette, IN 47907, USA

e-mail: dws@purdue.edu
Keywords Response congruency · Categorization · Memory retrieval $\cdot$ Response selection $\cdot$ Task switching

Task-switching research has generally focused on the performance of well-practiced tasks involving frequently repeated target stimuli and fixed response mappings (for reviews, see Kiesel et al., 2010; Vandierendonck, Liefooghe, \& Verbruggen, 2010). Practice effects have been analyzed in some studies (e.g., Buchler, Hoyer, \& Cerella, 2008; Logan \& Schneider, 2006; Minear \& Shah, 2008), but rarely at the level of individual target presentations to assess item-specific learning (Waszak, Hommel, \& Allport, 2003), even though such learning could influence how responses are selected in ambiguous task-switching situations. In the present study, we investigated the time course of learning for individual targets in a task-switching context to achieve insight regarding the development and characteristics of a nonmediated route for response selection.

A distinction can be made between two routes for response selection in task-switching situations: a mediated route and a nonmediated route (Kiesel, Wendt, \& Peters, 2007; Meiran \& Kessler, 2008; Schneider, 2014, 2015; Schneider \& Logan, 2009, 2014). The mediated route involves categorizing a target with respect to one or more tasks, then using the instructed category-response mappings to choose a response. The route is mediated because the intermediate step of categorization is performed (i.e., target $\rightarrow$ category $\rightarrow$ response). The nonmediated route involves using a target to retrieve a response directly from long-term memory following instance-based learning of target-response associations during practice (Logan, 1988, 2002). The route is nonmediated because the intermediate step of categorization is omitted (i.e., target $\rightarrow$ response). If a target's categories are mapped to different responses or a target is directly associated with 
different responses, then a task cue is needed to resolve the ambiguity and enable selection of a task-appropriate response.

Evidence supporting the existence of the mediated and nonmediated routes has come from studies of response congruency effects in task-switching performance (for an overview, see Schneider, 2015). To understand response congruency, consider an experiment in which origin (living or nonliving) and size (small or large) judgments are performed on the referents of target words (e.g., mosquito, camel, dime, boulder). If the two categories for each task are mapped to different response keys, with both tasks involving the same pair of keys (e.g., living and small mapped to a left key; nonliving and large mapped to a right key; see Fig. 1), then some targets will be congruent and require the same response regardless of the task (e.g., mosquito requires a left keypress response), and other targets will be incongruent and require different responses depending on the task (e.g., camel requires left and right keypress responses for the origin and size tasks, respectively). The response congruency effect is the finding of better performance for congruent than for incongruent targets (e.g., Brown, Reynolds, \& Braver, 2007; Kiesel et al., 2007; Meiran \& Kessler, 2008; Monsell, Sumner, \& Waters, 2003; Schneider, 2014, 2015; Schneider \& Logan, 2009, 2014; Sudevan \& Taylor, 1987). The effect can arise from the mediated route if response selection is facilitated when the same response is activated by congruent target categories, and impaired when different responses are activated by incongruent target categories. The effect can arise from the nonmediated

\section{Training}

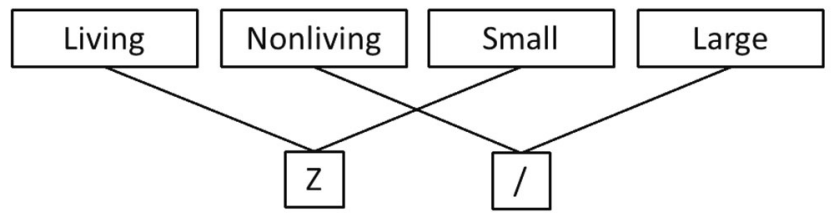

\section{Transfer (Experiment 1)}

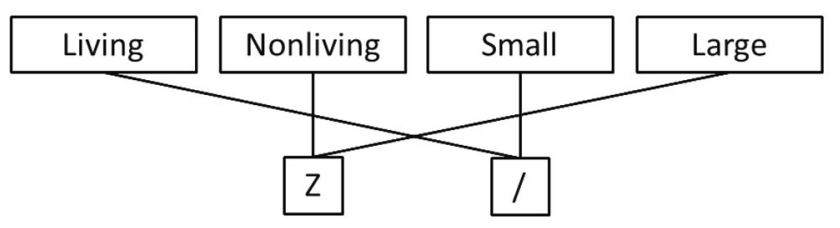

Transfer (Experiment 2)

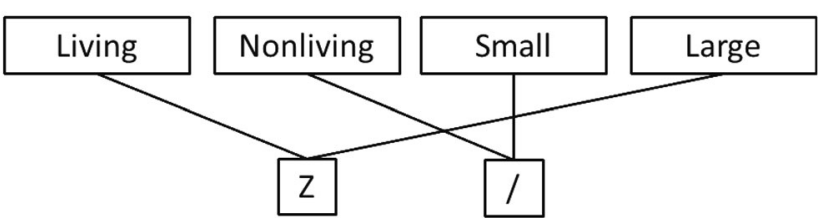

Fig. 1 Examples of category-response mappings for the training and transfer phases route if response selection is facilitated when a unique response is retrieved by a congruent target, and impaired when conflicting responses are retrieved by an incongruent target.

The existence of the mediated route has been supported by observations of response congruency effects in situations in which target-response instances are not available for retrieval, leaving categorization as the only mechanism for response selection. These findings include response congruency effects with nonrepeated targets (Schneider, 2015), unpracticed target-response mappings (Liefooghe, De Houwer, \& Wenke, 2013; Liefooghe, Wenke, \& De Houwer, 2012; but see Waszak, Wenke, \& Brass, 2008), and irrelevant distractors that never serve as targets (Reisenauer \& Dreisbach, 2013). Related findings have been reported by Wenke, Gaschler, and Nattkemper (2007) and, in the context of the flanker compatibility effect, by Cohen-Kdoshay and Meiran (2007, 2009). The existence of the nonmediated route has been supported by observations of inverted response congruency effects in situations in which category-response mappings are reversed for one task but not for the other, switching the response congruency of targets (Waszak, Pfister, \& Kiesel, 2013; Wendt \& Kiesel, 2008). However, additional evidence for the nonmediated route has been scarce, leaving unanswered questions about its development and characteristics.

We focused on two questions about the nonmediated route in the present study. First, what is the time course of learning for the nonmediated route? It is unclear how quickly the route can become functional as target-response instances accumulate in memory because response congruency effects have not been reported at the level of individual target presentations. Second, how are responses represented in the nonmediated route? It is unclear whether responses are represented in target-response instances at the specific level of manual keypresses (e.g., left or right keypress) or at the more abstract level of response congruency. The aforementioned findings of inverted response congruency effects with reversed category-response mappings (Waszak et al., 2013; Wendt \& Kiesel, 2008) cannot be used to distinguish between those possibilities in the absence of a comparison with a situation in which category-response mappings change but response congruency does not.

We conducted two task-switching experiments to answer these questions, as well as a question about the extent to which advance target processing can occur in task-switching situations. A notable aspect of both the mediated and the nonmediated routes is that responding to a congruent target does not necessarily entail exclusive performance of the relevant task on a trial (Schneider, 2015). The mediated route selects a response by categorizing the target with respect to both tasks (relevant and irrelevant), whereas the nonmediated route selects a response by direct memory retrieval, which does not require processing with respect to either experimentally 
defined task. Consequently, it is logically possible to select a response for a congruent target before the relevant task is known. This raises an important question: When given the opportunity, do subjects engage in the advance target processing afforded by the mediated and nonmediated routes?

This question cannot be answered using the typical cued task-switching procedure of presenting the task cue before the target (cue-target order; for a review, see Meiran, 2014). However, advance target processing can be investigated by presenting the target before the cue (target-cue order). This reversal of the stimulus order is rare in contemporary taskswitching research (Ruge, Braver, \& Meiran, 2009; Schneider \& Logan, 2014), but the rarity of a procedural variation does not imply that it is uninformative, as has been revealed by earlier research. In some of the first studies of cued task switching, Shaffer $(1965,1966)$ compared performance with cue-target and target-cue orders, finding that presenting either the cue or the target first led to better performance than did simultaneous presentation. Interestingly, taskswitch costs (slower performance for task switches than for task repetitions) were not affected by stimulus order, leading Shaffer to suggest that the benefit of advance stimulus processing was to reduce the contribution of stimulus encoding time to overall response time (RT). Related findings and conclusions were reported in subsequent studies (Bernstein \& Segal, 1968; Biederman, 1973; Davis \& Taylor, 1967). Biederman's study of mental arithmetic, in which the arithmetic operator (cue) was presented before or after the numerical operands (target), is particularly relevant in the present context because he obtained evidence suggesting that subjects precomputed the answers for both possible arithmetic operators when the targets were presented first, which is the kind of dualtask processing associated with the mediated route for response selection. Thus, the results of these studies suggest that advance target processing could progress from stimulus encoding to preliminary response selection in some contexts, a conclusion made possible by investigating target-cue order.

Advance target processing was still limited in previous studies that involved target-cue order because the targets were often incongruent, making the cues useful and necessary for accurate performance (Kantowitz \& Sanders, 1972). This limitation was absent in a recent study of ours (Schneider \& Logan, 2014) in which performance with cue-target and target-cue orders was assessed for both incongruent and congruent targets (see also Ruge et al., 2009). The results revealed faster performance with both stimulus orders than with simultaneous presentation, replicating previous findings (Bernstein \& Segal, 1968; Biederman, 1973; Davis \& Taylor, 1967; Shaffer, 1965, 1966). Critically, we found response congruency effects for both stimulus orders, implicating either the mediated or the nonmediated route (or both) in performance. These results were obtained regardless of whether the stimulus orders were presented in separate blocks or intermixed within the same blocks of trials, suggesting that target-cue order did not induce a markedly different processing strategy than did cue-target order, but not implying that processing was wholly equivalent. Evidence of advance target processing was provided by three aspects of the data from the target-cue order trials. First, RT became shorter as the stimulus onset asynchrony (SOA) became longer, suggesting that some target encoding occurred during the SOA. Second, the response congruency effect on RT increased with SOA (primarily reflecting a speedup in responding for congruent targets), suggesting that targets were processed to the level of response selection during the SOA. Third, at the longest SOA used (800 ms), subjects responded to congruent targets prior to cue onset on over $35 \%$ of trials, which represented unequivocal evidence that advance target processing involved response selection.

These results indicated that our subjects engaged in advance target processing when given the opportunity to do so, but it remained unclear whether the mediated or the nonmediated route was primarily responsible for early response selection. In Schneider and Logan (2014), we showed that a computational model of the mediated route (compound cue retrieval; Schneider \& Logan, 2005, 2009) could account for major features of our data when the mediated route was allowed to function with only one stimulus, but those modeling results did not exclude a potential contribution of the nonmediated route, which we suggested would have to be integrated with the mediated route to develop a more comprehensive model of response selection in task switching. Indeed, given that the nonmediated route does not depend on processing the target with respect to either task signaled by the cue, we contend that it may be more likely than the mediated route to generate responses to congruent targets prior to cue onset, but only after enough target-response instances have accumulated in memory from practice. Thus, it is possible that practice-related changes in early response selection for congruent targets can provide insight regarding the time course of learning for the nonmediated route, thereby helping to answer one of the questions about the nonmediated route that motivated the present study.

Our experiments had four features that allowed us to examine the development and characteristics of the nonmediated route. First, we measured performance at the level of individual target presentations, which enabled fine-grained observation of practice effects and calculation of response congruency effects for each target presentation. Note that a response congruency effect for the first target presentation would represent evidence of the mediated route and replicate Schneider's (2015) results with nonrepeated targets. Changes in the response congruency effect with practice would largely represent the initiation and increasing involvement of the nonmediated route. Second, after a training phase in which practice effects were established, we had a transfer phase in which the category-response mappings changed (as described 
below), which provided a way to examine the nature of the response representation in memory and to replicate Wendt and Kiesel's (2008) results (in Experiment 2). Third, we introduced new targets in the transfer phase, which enabled comparisons with old targets to determine whether practice and transfer effects were generic or target-specific. Fourth, we presented the target before the task cue on every trial, which provided a third dependent measure besides RT and error rate: the percentage of early responses, $p$ (early), which are responses occurring prior to cue onset. Used previously by Schneider and Logan (2014) and alluded to earlier, $p$ (early) is informative because congruent targets afford response selection and execution before the relevant task is known; therefore, $p$ (early) can be used to measure the extent of advance target processing irrespective of the task-switching circumstances, potentially yielding additional evidence regarding the development of the nonmediated route.

\section{Experiment 1}

Experiment 1 involved switching between origin (living or nonliving) and size (small or large) tasks performed on target words. Same-task categories were mapped to different response keys, but the categories mapped to each response during training were remapped to the alternative response during transfer (see Fig. 1). Consequently, all category-response mappings changed, but response congruency for a given target was maintained from training to transfer. The target was presented before the cue on every trial to provide an opportunity for advance target processing. The questions of interest were how quickly the nonmediated route would be learned and whether its functionality would be disrupted by the changed category-response mappings, providing insight regarding the nature of the response representation.

\section{Method}

Subjects Twenty-four students from Vanderbilt University participated for course credit.

Apparatus, tasks, and stimuli Computers displayed stimuli on monitors and registered responses from QWERTY keyboards. The stimuli were displayed in white 12-point Courier New font on a black background, viewed at a distance of about $50 \mathrm{~cm}$. The tasks were origin and size judgments of the referents of target words. The origin task involved categorizing a target as living or nonliving and was cued by the word ORIGIN or LIFE. The size task involved categorizing a target as small or large (relative to the size of a basketball) and was cued by the word SIZE or MAGNITUDE. We used two cues per task to maintain continuity with our previous work (e.g., Schneider \& Logan, 2014). The targets were 64 words divided equally into two sets, with eight targets for each category combination in each set (see Appendix A). The mean word length was 5.7 letters and the mean word frequency was 3.1 occurrences per million. Separate 2 (set) $\times 2$ (origin) $\times 2$ (size) analyses of variance (ANOVAs) on each variable revealed no significant effects, all $F_{\mathrm{S}}<1$.

Procedure Subjects sat at computers in individual testing rooms after providing informed consent for a study protocol approved by the Vanderbilt University Institutional Review Board. Instructions were presented onscreen and explained by the experimenter. The experiment was divided into training and transfer phases, both involving the same trial procedure. Each trial began with two vertically arranged fixation crosses presented centrally onscreen. After $500 \mathrm{~ms}$, both crosses disappeared and a target was presented. After an additional $800 \mathrm{~ms}$, a cue was presented. The cue and target appeared at the previous locations of the top and bottom crosses, respectively. Subjects were informed that a response was allowed any time after target onset. If they responded before or less than $250 \mathrm{~ms}$ after cue onset, the trial continued until the cue was presented and had been displayed for $250 \mathrm{~ms}$, and then the screen was cleared. If they responded more than $250 \mathrm{~ms}$ after cue onset, then the screen was cleared immediately after the response. Thus, regardless of when a response occurred, both the cue and the target were presented on each trial. After the screen was cleared, it remained blank for $500 \mathrm{~ms}$, and then the fixation display for the next trial was presented. Responses were made with the "Z" and "/" keys on the keyboard, with same-task categories mapped to different keys (see Fig. 1) and all possible category-response mappings counterbalanced across subjects. Reminders of the mappings appeared in the bottom corners of the screen.

In the training phase, subjects completed mini-blocks of 32 trials involving the targets from one set (the sets were counterbalanced across subjects). Cues and targets were randomly selected in each mini-block, with each cue occurring eight times and each target occurring once. There were 20 mini-blocks (640 trials); therefore, each target was presented 20 times during training. A rest period occurred after every two mini-blocks, which together represented a single, uninterrupted block from the subject's perspective. In the transfer phase, subjects received instructions about changed categoryresponse mappings: The categories mapped to each response during training were remapped to the alternative response during transfer. For example, if a subject had pressed the "Z" key for living or small and the "/" key for nonliving or large during training, then he or she would press the " $Z$ " key for nonliving or large and the "/" key for living or small during transfer (see Fig. 1). Thus, all category-response mappings changed, but response congruency was maintained from training to transfer (i.e., a congruent target in training was congruent in transfer, and an incongruent target in training was incongruent in transfer). The onscreen reminders of the 
mappings changed appropriately. Subjects were informed that new targets would be mixed among the old targets in the transfer phase, which involved blocks of 64 trials with the targets from both sets. Old targets were those from the set used in training, and new targets were those from the unused set. Targets were randomly selected in each block, with each target occurring once. There were four blocks (256 trials); therefore, each target was presented four times during transfer. A rest period occurred after every block.

\section{Results and discussion}

RT was defined relative to target onset. We excluded trials with RTs longer than $4,000 \mathrm{~ms}$ from all analyses (1.5\% of trials) and excluded error trials from the RT analysis. We included trials with responses that occurred before cue presentation in the RT and error analyses because there were many empty cells when response type (before or after cue presentation) was included as an additional variable in the analyses or when we restricted the analyses to trials with responses that occurred after cue presentation only. The empty cells occurred because several subjects did not respond before the cue on any trial early in training, whereas several subjects responded before the cue on every congruent trial late in training. We included both correct and error trials in the $p$ (early) analysis because the results did not change materially when error trials were excluded.

We focused our analyses on response congruency effects because they were most relevant for answering our questions of interest. We collapsed over task transition (task switch or task repetition) because the results indicated that task transition effects were uninterpretable. More specifically, we obtained evidence that response selection on many trials involved either categorizing the target with respect to both tasks (via the mediated route) or performing neither task and instead using the target to retrieve an associated response from memory (via the nonmediated route). Thus, it could not be determined whether cognitive processing on any given trial reflected the nominal task transition (for further discussion, see Schneider \& Logan, 2014). On a more practical note, fewer than 10 observations would have contributed to each condition mean per subject if task transition had been included as an additional variable in the data analyses.

Training Training effects were evaluated by analyzing data from the first 20 target presentations. Mean RTs, $p$ (early), and error rates were submitted to repeated measures ANOVAs with Response Congruency and Target Presentation Number as factors. Note that the target presentation number is equivalent to the training mini-block number because each target occurred once per mini-block. The ANOVA results are summarized in Table 1, which indicates that all effects were significant for RT and $p$ (early). The mean error rate was $4.5 \%$, and there was a response congruency effect of $5.2 \%$, which represented the only significant effect on error rate.

Mean RTs are plotted as a function of response congruency and target presentation number in Fig. 2. To aid comparisons with the $p$ (early) data, the response congruency effect is plotted as a function of target presentation number in Fig. 3. A reliable response congruency effect can be seen starting with the first target presentation, $t(23)=3.14, S E=55, p<.05$, replicating Schneider's (2015) results with nonrepeated targets. Without target-response instances to retrieve from memory at this point, this effect represents evidence that the mediated route was used to select responses early in training. RTs became shorter as the number of presentations increased (see Fig. 2), but this change was greater for congruent than for incongruent targets, resulting in an increase in the response congruency effect with practice (see Fig. 3). The speedup for incongruent targets can be attributed to improvements in target encoding and categorization, resulting in a more efficient mediated route. The same improvements likely occurred for congruent targets, but the more pronounced speedup for congruent targets suggests the initiation and increasing involvement of the nonmediated route for response selection. With practice, a congruent target was mapped repeatedly to the same response, making the association represented more strongly in memory and speeding retrieval (Logan, 1988, 2002). Consistent with previous results involving word categorization (Logan \& Etherton, 1994), the speedup for congruent targets seemed to reach asymptote after 15 presentations. A secondary analysis of the RT data that focused on sequential effects involving response congruency is reported in Appendix B.

The changes in the response congruency effect on RT were paralleled by changes in $p$ (early), which is plotted as a function of response congruency and target presentation number in Fig. 4. There was an increase in $p$ (early) for congruent targets with practice, but little change in the low $p$ (early) level for incongruent targets (see Fig. 4). The finding that subjects responded before cue presentation on some trials replicates Schneider and Logan (2014) and indicates that subjects engaged in advance target processing until they had completed response selection, which was not contingent on knowing the relevant task. Consequently, cognitive processing did not reflect the nominal task transition on many trials, making any task transition effects uninterpretable. The rise in $p$ (early) for congruent targets was strongly correlated with the increase in the response congruency effect on RT $(r=.99)$, suggesting that the nonmediated route dominated response selection by the end of training, but not ruling out a continued contribution of the mediated route. ${ }^{1}$

\footnotetext{
${ }^{1}$ Even though early responses contributed strongly to changes in the response congruency effect during training, we still observed numerical increases in the response congruency effect on RT with practice in Experiments 1 and 2 when we recalculated the means only for trials with responses that occurred after cue presentation.
} 
Table 1 Summary of analyses of variance on the training data for Experiment 1

\begin{tabular}{|c|c|c|c|c|c|c|c|c|c|c|}
\hline \multirow[b]{2}{*}{ Effect } & \multirow[b]{2}{*}{$d f$} & \multicolumn{3}{|c|}{ Response Time } & \multicolumn{3}{|l|}{$p$ (early) } & \multicolumn{3}{|c|}{ Error Rate } \\
\hline & & $F$ & MSE & $\eta_{\mathrm{p}}^{2}$ & $F$ & $M S E$ & $\eta_{\mathrm{p}}^{2}$ & $F$ & $M S E$ & $\eta_{\mathrm{p}}^{2}$ \\
\hline Response congruency (C) & 1,23 & $134.05^{*}$ & 608,695 & .85 & $101.98^{*}$ & 4,937 & .82 & $25.86^{*}$ & 250 & .53 \\
\hline Presentation $(\mathrm{P})$ & 19,437 & $85.63^{*}$ & 46,006 & .79 & $44.22^{*}$ & 200 & .66 & 0.51 & 28 & .02 \\
\hline $\mathrm{C} \times \mathrm{P}$ & 19,437 & $21.36^{*}$ & 26,309 & .48 & $40.87^{*}$ & 170 & .64 & 1.30 & 31 & .05 \\
\hline
\end{tabular}

Transfer Transfer effects were evaluated in two sets of analyses. The first analysis compared performance for the last training presentation and the first transfer presentation of old targets to assess the effects of the changed category-response mappings. Mean RTs, $p$ (early), and error rates were submitted to repeated measures ANOVAs with Response Congruency and Phase (training or transfer) as factors. The ANOVA results are summarized in Table 2, which indicates that nearly all effects were significant for RT and $p$ (early). The mean error rate was $4.9 \%$, and there was a response congruency effect of $2.7 \%$, which represented the only significant effect on error rate.

Mean RT became longer for old targets from training to transfer (see Fig. 2), but the changes were similar for congruent and incongruent targets, resulting in little change to the response congruency effect (see Fig. 3). Consistent with the RT data, $p$ (early) decreased initially from training to transfer (see Fig. 4). The modest performance decrement and stability of the response congruency effect from training to transfer suggest that the target-response instances underlying the nonmediated route involve relatively abstract response representations with information about response congruency. That

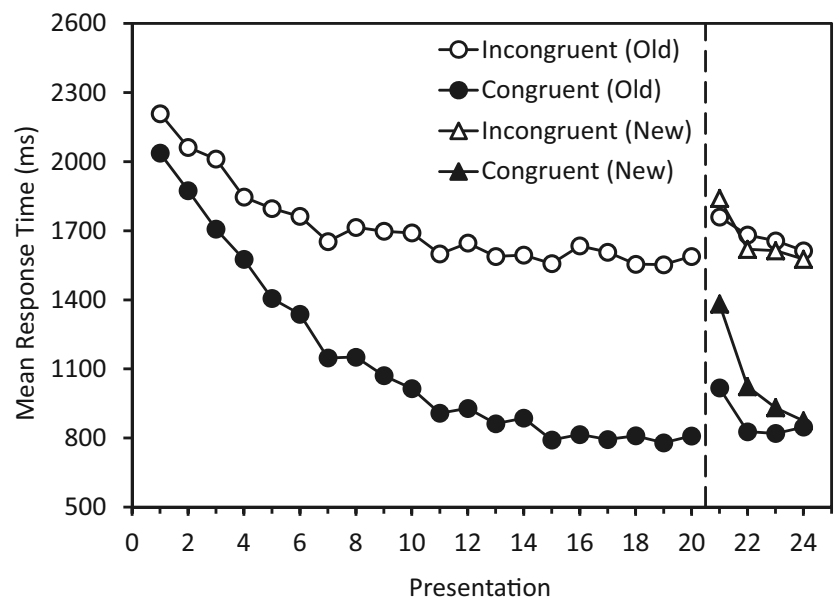

Fig. 2 Mean response times as a function of response congruency, target type (old or new), and target presentation number in Experiment 1. The broken vertical line represents the transition from training to transfer. For old targets, response congruency was maintained from training to transfer. For new targets, the presentations numbered 21-24 were actually Presentations 1-4 is, even though the manual response to a given target changed from training to transfer, the mapping to an abstract response label (e.g., "same" or "different" responses across tasks) did not. The results suggest that subjects could rapidly remap this response congruency information to a different manual response without much difficulty.

The second analysis of transfer effects involved evaluating performance for old and new targets to assess the effects of target experience. Mean RTs, $p$ (early), and error rates were submitted to repeated measures ANOVAs with Response Congruency, Target Type (old or new), and Target Presentation Number (1-4) as factors. Note that the target presentation number is equivalent to the transfer block number because each target occurred once per block. The ANOVA results are summarized in Table 3, which indicates that all effects were significant for RT and $p$ (early). The mean error rate was $5.4 \%$, and the response congruency effect of $2.2 \%$ was marginally significant $(p=.07)$.

RTs were similar for new and old incongruent targets, whereas RTs were longer for new than for old congruent targets (see Fig. 2). This difference decreased during transfer, resulting in the response congruency effect for new targets

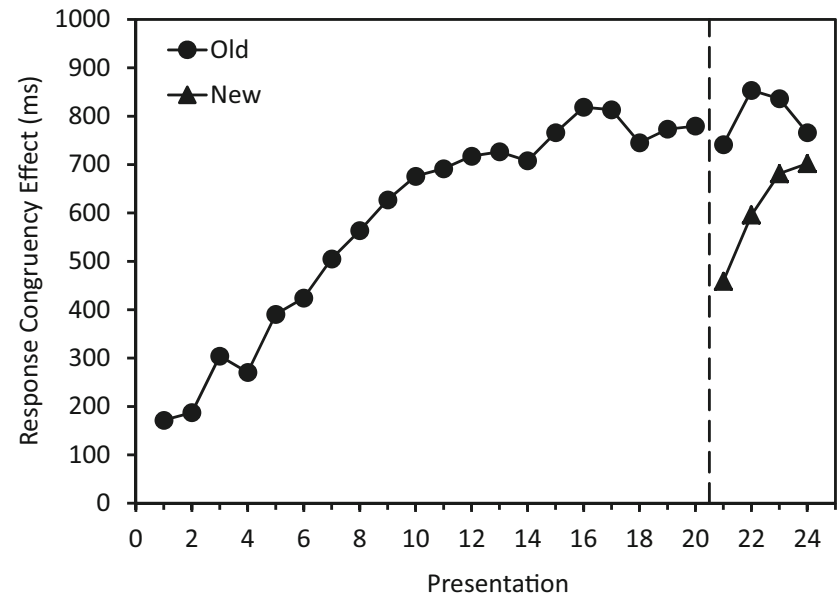

Fig. 3 Response congruency effects on response time as a function of target type (old or new) and target presentation number in Experiment 1. The broken vertical line represents the transition from training to transfer. For old targets, response congruency was maintained from training to transfer. For new targets, the presentations numbered 21-24 were actually Presentations 1-4 


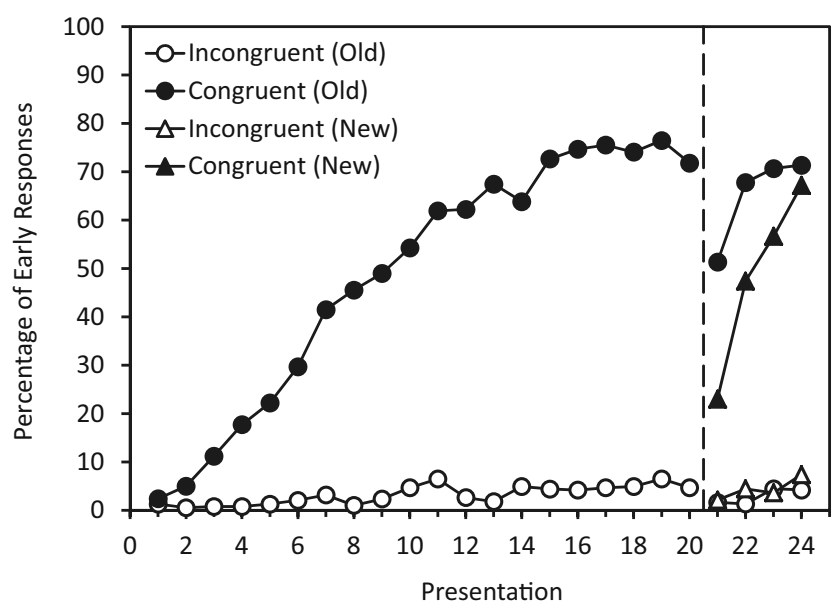

Fig. 4 Percentages of early responses, $p$ (early), as a function of response congruency, target type (old or new), and target presentation number in Experiment 1 . The broken vertical line represents the transition from training to transfer. For old targets, response congruency was maintained from training to transfer. For new targets, the presentations numbered 21-24 were actually Presentations 1-4

approaching the magnitude of the effect for old targets (see Fig. 3). Consistent with the RT data, $p$ (early) did not differ for new and old incongruent targets during transfer, whereas it was lower for new than for old congruent targets (see Fig. 4). This difference decreased during transfer until the $p$ (early) values were nearly equivalent for new and old congruent targets. The similar results for new and old incongruent targets suggest that response selection for those targets was based primarily on the mediated route, which remained functional regardless of whether targets had been experienced. Moreover, the results are consistent with the idea that the speedup for incongruent targets during training was partly due to a general improvement in categorization, which would benefit both old and new incongruent targets during transfer and explain why the starting point for performance with new incongruent targets in transfer was better than the starting point for incongruent targets in training. The different results for new and old congruent targets suggest that response selection for those targets was based primarily on the nonmediated route, which benefited from prior experience with the old targets. However, the starting point and rapid improvement in performance for new congruent targets raise the possibility that target-response associations for new targets can be learned quickly once the nonmediated route is active, perhaps reflecting a strategic adjustment by subjects.

\section{Experiment 2}

Experiment 2 was a replication of Experiment 1, except in terms of how category-response mappings changed from training to transfer. Only one category (instead of both) from the pair mapped to each response during training was remapped to the alternative response during transfer (see Fig. 1). Consequently, the category-response mappings changed for one task and not for the other, resulting in response congruency being reversed from training to transfer (as in Wendt \& Kiesel, 2008). Given that Experiment 1 suggested that the target-response instances underlying the nonmediated route involve response labels coding response congruency ("same" or "different"), we predicted that the nonmediated route's functionality would be disrupted by reversing those labels with the changed category-response mappings in the transfer phase of Experiment 2.

Method

Subjects Thirty-two students from Vanderbilt University participated for course credit. None of them had participated in Experiment 1.

Apparatus, tasks, and stimuli These were identical to the same aspects of Experiment 1.

Procedure The procedure was identical to that of Experiment 1 except for how the category-response mappings changed in the transfer phase. Only one category (instead of both) from the pair mapped to each response during training was remapped to the alternative response during transfer. For example, if a subject pressed the "Z" key for living or small and the "/" key for nonliving or large during training, then he or she might press the "Z" key for living or large and the "/" key for nonliving or small during transfer (see Fig. 1). Thus, the category-response mappings changed for one task and not for

Table 2 Summary of analyses of variance on the training-to-transfer data for Experiment 1

\begin{tabular}{|c|c|c|c|c|c|c|c|c|c|c|}
\hline \multirow[b]{2}{*}{ Effect } & \multirow[b]{2}{*}{$d f$} & \multicolumn{3}{|c|}{ Response Time } & \multicolumn{3}{|l|}{$p$ (early) } & \multicolumn{3}{|c|}{ Error Rate } \\
\hline & & $F$ & $M S E$ & $\eta_{\mathrm{p}}{ }^{2}$ & $F$ & $M S E$ & $\eta_{\mathrm{p}}^{2}$ & $F$ & $M S E$ & $\eta_{\mathrm{p}}{ }^{2}$ \\
\hline Response congruency $(\mathrm{C})$ & 1,23 & $184.49^{*}$ & 75,240 & .89 & $148.04^{*}$ & 553 & .87 & $5.62^{*}$ & 32 & .20 \\
\hline Phase $(\mathrm{P})$ & 1,23 & $26.57^{*}$ & 32,389 & .54 & $27.67^{*}$ & 120 & .55 & 0.76 & 22 & .03 \\
\hline $\mathrm{C} \times \mathrm{P}$ & 1,23 & 0.41 & 21,520 & .02 & $15.97^{*}$ & 114 & .41 & 3.57 & 44 & .13 \\
\hline
\end{tabular}

${ }^{*} p<.05$ 
Table 3 Summary of analyses of variance on the transfer data for Experiment 1

\begin{tabular}{|c|c|c|c|c|c|c|c|c|c|c|}
\hline \multirow[b]{2}{*}{ Effect } & \multirow[b]{2}{*}{$d f$} & \multicolumn{3}{|c|}{ Response Time } & \multicolumn{3}{|l|}{$p$ (early) } & \multicolumn{3}{|c|}{ Error Rate } \\
\hline & & $F$ & $M S E$ & $\eta_{\mathrm{p}}{ }^{2}$ & $F$ & $M S E$ & $\eta_{\mathrm{p}}{ }^{2}$ & $F$ & $M S E$ & $\eta_{\mathrm{p}}{ }^{2}$ \\
\hline Response congruency (C) & 1,23 & $164.30^{*}$ & 289,982 & .88 & $158.35^{*}$ & 1,721 & .87 & 3.69 & 127 & .14 \\
\hline Target type $(\mathrm{T})$ & 1,23 & $33.80^{*}$ & 18,001 & .60 & $46.82^{*}$ & 119 & .67 & 3.34 & 51 & .13 \\
\hline Presentation (P) & 3,69 & $37.58^{*}$ & 39,065 & .62 & $35.67^{*}$ & 163 & .61 & 0.99 & 31 & .04 \\
\hline $\mathrm{C} \times \mathrm{T}$ & 1,23 & $29.27^{*}$ & 29,551 & .56 & $67.69^{*}$ & 118 & .75 & $35.71^{*}$ & 34 & .61 \\
\hline $\mathrm{C} \times \mathrm{P}$ & 3,69 & $8.37^{*}$ & 14,460 & .27 & $38.19^{*}$ & 98 & .62 & 0.42 & 40 & .02 \\
\hline $\mathrm{T} \times \mathrm{P}$ & 3,69 & $17.71^{*}$ & 13,397 & .44 & $8.52^{*}$ & 86 & .27 & $2.82^{*}$ & 23 & .11 \\
\hline $\mathrm{C} \times \mathrm{T} \times \mathrm{P}$ & 3,69 & $3.34^{*}$ & 18,126 & .13 & $6.33^{*}$ & 90 & .22 & $3.17^{*}$ & 27 & .12 \\
\hline
\end{tabular}

${ }^{*} p<.05$

the other, resulting in response congruency being reversed from training to transfer (i.e., a congruent target in training became incongruent in transfer, and an incongruent target in training became congruent in transfer). The task for which the category-response mappings changed was counterbalanced across subjects.

\section{Results and discussion}

As in Experiment 1, we excluded trials with RTs longer than 4, $000 \mathrm{~ms}$ from all analyses (1.1\% of trials) and excluded error trials from the RT analysis. We included trials with responses that occurred before cue presentation in the RT and error analyses for the reasons described earlier. We included correct and error trials in the $p$ (early) analysis because the results did not change materially when error trials were excluded.

Training Training effects were evaluated by analyzing data from the first 20 target presentations. Mean RTs, $p$ (early), and error rates were submitted to repeated measures ANOVAs with Response Congruency and Target Presentation Number as factors. The ANOVA results are summarized in Table 4, which indicates that all effects were significant for RT and $p$ (early). The mean error rate was $4.4 \%$, and there was a response congruency effect of $5.9 \%$, which represented the only significant effect on error rate.
Mean RTs are plotted as a function of response congruency and target presentation number in Fig. 5. The response congruency effect is plotted as a function of target presentation number in Fig. 6. We found a reliable response congruency effect starting with the first target presentation, $t(31)=4.22$, $S E=53, p<.05$, replicating Experiment 1 and Schneider (2015). This effect represents further evidence that the mediated route was used to select responses early in training. RTs became shorter as the number of presentations increased (see Fig. 5), but this change was greater for congruent targets (which seemed to reach asymptote after 15 presentations) than for incongruent targets, resulting in an increase in the response congruency effect with practice (see Fig. 6). This pattern of improved performance replicates Experiment 1 and suggests that the nonmediated route was increasingly used to select responses for congruent targets as training progressed. A secondary analysis of the RT data that focused on sequential effects involving response congruency is reported in Appendix B.

The changes in the response congruency effect on RT were paralleled by changes in $p$ (early), which is plotted as a function of response congruency and target presentation number in Fig. 7. There was an increase in $p$ (early) for congruent targets with practice, but little change in the low $p$ (early) level for incongruent targets (see Fig. 7). The advance responding that occurred on some trials replicates Experiment 1 and Schneider and Logan (2014), providing additional evidence that subjects engaged in advance target processing when given the

Table 4 Summary of analyses of variance on the training data for Experiment 2

\begin{tabular}{|c|c|c|c|c|c|c|c|c|c|c|}
\hline \multirow[b]{2}{*}{ Effect } & \multirow[b]{2}{*}{$d f$} & \multicolumn{3}{|c|}{ Response Time } & \multicolumn{3}{|l|}{$p$ (early) } & \multicolumn{3}{|c|}{ Error Rate } \\
\hline & & $F$ & $M S E$ & $\eta_{\mathrm{p}}^{2}$ & $F$ & $M S E$ & $\eta_{\mathrm{p}}{ }^{2}$ & $F$ & $M S E$ & $\eta_{\mathrm{p}}^{2}$ \\
\hline Response congruency (C) & 1,31 & $686.14^{*}$ & 215,058 & .96 & $388.76^{*}$ & 2,688 & .93 & $97.14^{*}$ & 113 & .76 \\
\hline Presentation $(\mathrm{P})$ & 19,589 & $78.05^{*}$ & 53,330 & .72 & $72.48^{*}$ & 168 & .70 & 1.36 & 26 & .04 \\
\hline $\mathrm{C} \times \mathrm{P}$ & 19,589 & $34.62^{*}$ & 24,606 & .53 & $77.52^{*}$ & 151 & .71 & 1.10 & 26 & .03 \\
\hline
\end{tabular}

${ }^{*} p<.05$ 


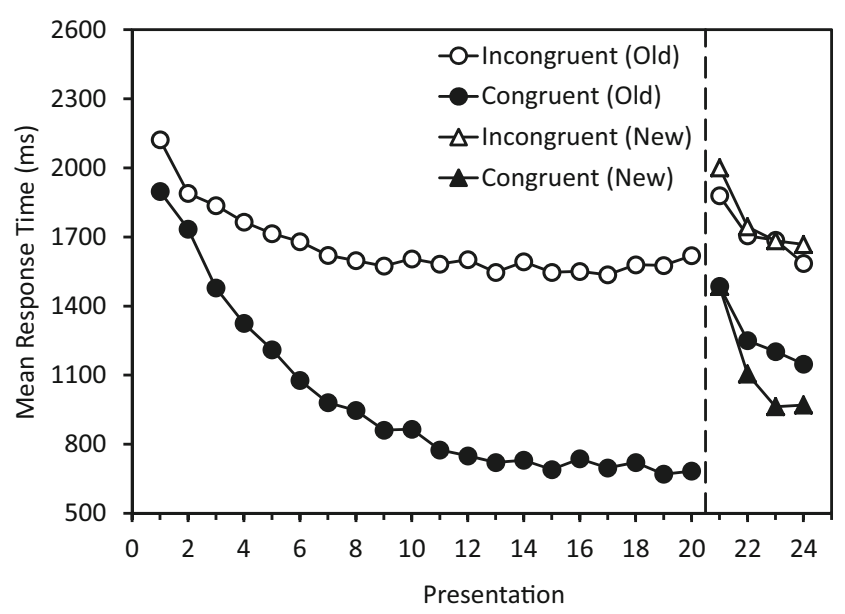

Fig. 5 Mean response times as a function of response congruency, target type (old or new), and target presentation number in Experiment 2. The broken vertical line represents the transition from training to transfer. For old targets, response congruency was reversed from training to transfer, such that the old targets labeled "incongruent" in transfer correspond to the congruent targets in training, and the old targets labeled "congruent" in transfer correspond to the incongruent targets in training. For new targets, the presentations numbered 21-24 were actually Presentations $1-4$

opportunity to do so. The rise in $p$ (early) for congruent targets was strongly correlated with the increase in the response congruency effect on RT $(r=.99)$, consistent with the results of Experiment 1.

Transfer Transfer effects were evaluated in two sets of analyses. The first analysis compared performance for the last training presentation and the first transfer presentation of old

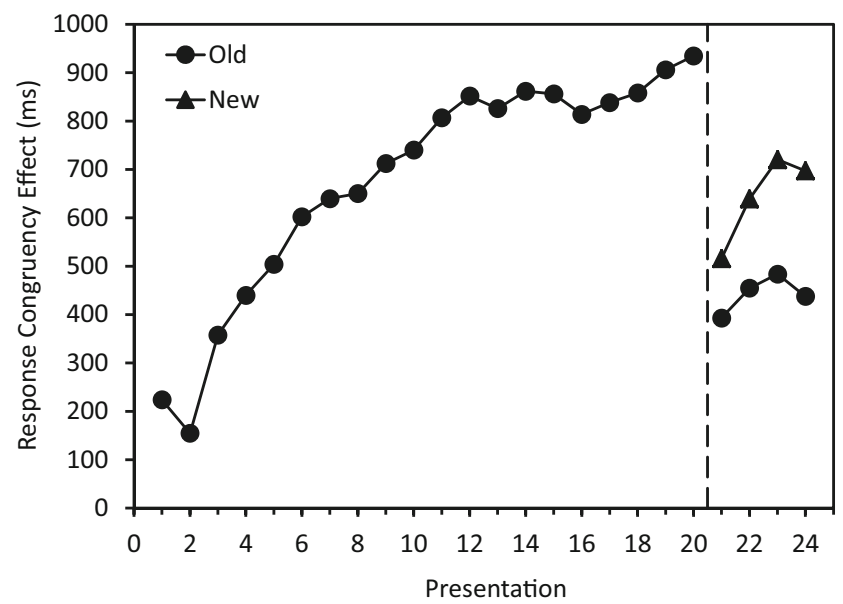

Fig. 6 Response congruency effects on response time as a function of target type (old or new) and target presentation number in Experiment 2. The broken vertical line represents the transition from training to transfer. For old targets, response congruency was reversed from training to transfer; therefore, what is shown for old targets is actually an inverted response congruency effect if the original response congruency labels from training are used. For new targets, the presentations numbered 2124 were actually Presentations 1-4

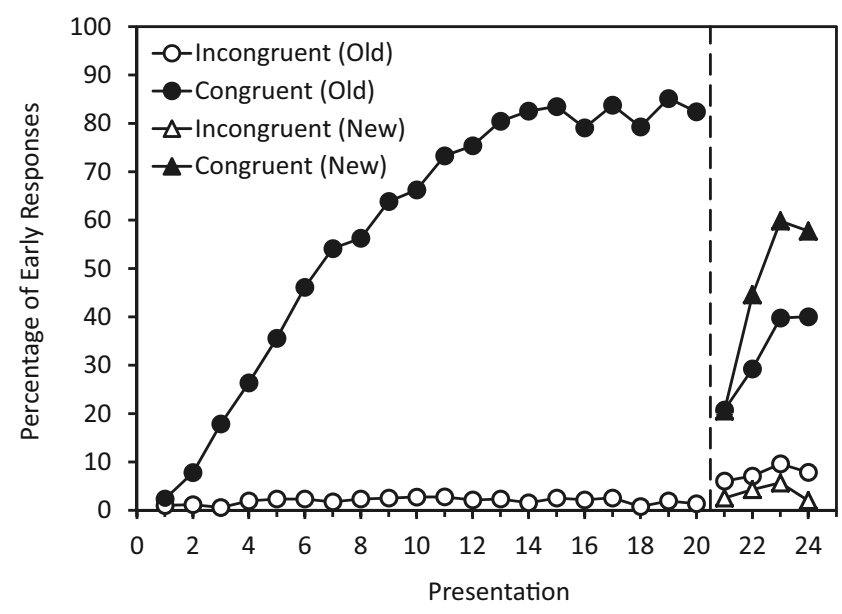

Fig. 7 Percentages of early responses, $p$ (early), as a function of response congruency, target type (old or new), and target presentation number in Experiment 2. The broken vertical line represents the transition from training to transfer. For old targets, response congruency was reversed from training to transfer, such that the old targets labeled "incongruent" in transfer correspond to the congruent targets in training, and the old targets labeled "congruent" in transfer correspond to the incongruent targets in training. For new targets, the presentations numbered 21-24 were actually Presentations $1-4$

targets to assess the effects of the changed category-response mappings. Mean RTs, $p$ (early), and error rates were submitted to repeated measures ANOVAs with Response Congruency and Phase as factors. The ANOVA results are summarized in Table 5, which indicates that all effects were significant for RT and $p$ (early). The mean error rate was $5.0 \%$, and there was a response congruency effect of $5.9 \%$, which represented the only significant effect on error rate.

Mean RT became longer for old targets from training to transfer (see Fig. 5), but the change was greater for congruent than for incongruent targets, resulting in a reduction in the response congruency effect (see Fig. 6). However, recall that response congruency was reversed from training to transfer. The labeling of the transfer data for old targets in Figs. 5 and 7 is based on the response congruency of those targets in the transfer phase, which means that the old targets labeled "incongruent" in transfer (white circles in the right regions of the figures) are the same words that had been congruent in training (black circles in the left regions of the figures), and the old targets labeled "congruent" in transfer (black circles in the right regions of the figures) are the same words that had been incongruent in training (white circles in the left regions of the figures). If we were to use the original response congruency labels from training, then Fig. 6 actually shows an inverted response congruency effect for old targets during transfer, replicating Wendt and Kiesel (2008; see also Waszak et al., 2013). The inversion in the RT data is also reflected in the $p$ (early) data. For old targets that changed from incongruent to congruent, $p$ (early) increased from $1.4 \%$ in 
Table 5 Summary of analyses of variance on the training-to-transfer data for Experiment 2

\begin{tabular}{|c|c|c|c|c|c|c|c|c|c|c|}
\hline \multirow[b]{2}{*}{ Effect } & \multirow[b]{2}{*}{$d f$} & \multicolumn{3}{|c|}{ Response Time } & \multicolumn{3}{|l|}{$p$ (early) } & \multicolumn{3}{|c|}{ Error Rate } \\
\hline & & $F$ & $M S E$ & $\eta_{\mathrm{p}}{ }^{2}$ & $F$ & $M S E$ & $\eta_{\mathrm{p}}{ }^{2}$ & $F$ & $M S E$ & $\eta_{\mathrm{p}}^{2}$ \\
\hline Response congruency (C) & 1,31 & $440.35^{*}$ & 32,038 & .93 & $407.13^{*}$ & 180 & .93 & $27.88^{*}$ & 40 & .47 \\
\hline Phase $(\mathrm{P})$ & 1,31 & $120.10^{*}$ & 75,218 & .79 & $101.10^{*}$ & 257 & .77 & 4.01 & 30 & .11 \\
\hline $\mathrm{C} \times \mathrm{P}$ & 1,31 & $75.74^{*}$ & 30,989 & .71 & $276.87^{*}$ & 127 & .90 & 0.08 & 27 & $<.01$ \\
\hline
\end{tabular}

training to $20.8 \%$ in transfer, whereas for old targets that changed from congruent to incongruent, $p$ (early) decreased from $82.4 \%$ in training to $6.1 \%$ in transfer. The inversion of the response congruency effect from training to transfer is consistent with the idea that the target-response instances underlying the nonmediated route involve response representations with information about response congruency ("same" or "different"). In contrast with Experiment 1, this information became invalid when category-response mappings changed in Experiment 2 (e.g., a target associated with a "same" response label in training had to be associated with a "different" response label in transfer). The results suggest that subjects were unable to immediately rewrite the response labels, which meant that the nonmediated route continued to retrieve now-inappropriate response labels that impaired performance for previously congruent targets, yielding an inverted response congruency effect.

The second analysis of transfer effects involved evaluating performance for old and new targets to assess the effects of target experience. Mean RTs, $p$ (early), and error rates were submitted to repeated measures ANOVAs with Response Congruency, Target Type, and Target Presentation Number as factors. The ANOVA results are summarized in Table 6, which indicates that nearly all effects were significant for RT and $p$ (early). The mean error rate was $5.3 \%$, and there was a response congruency effect of $5.9 \%$, which represented the only significant effect on error rate.
RTs were similar for old and new incongruent targets, whereas RTs were longer for old than for new congruent targets (see Fig. 5). This difference increased during transfer, resulting in the response congruency effect for new targets being larger than that for old targets (see Fig. 6). Consistent with the RT data, $p$ (early) did not differ much for old and new incongruent targets during transfer, whereas $p$ (early) was lower for old than for new congruent targets, a difference that increased during transfer (see Fig. 7). As in Experiment 1, the similar results for new and old incongruent targets suggest that response selection for those targets was based primarily on the mediated route, for which categorization may have become more efficient with practice. The different results for new and old congruent targets suggest different levels of consistency within the nonmediated route. For new congruent targets, memory contained a homogeneous set of target-response instances because those targets were experienced with only one set of category-response mappings, resulting in quick learning and response selection via the nonmediated route. For old congruent targets (which had been incongruent in training), memory contained a heterogeneous set of target-response instances because those targets were experienced with two sets of category-response mappings, resulting in slower (re)learning and response selection via the nonmediated route. The greater consistency within the nonmediated route for new

Table 6 Summary of analyses of variance on the transfer data for Experiment 2

\begin{tabular}{|c|c|c|c|c|c|c|c|c|c|c|}
\hline \multirow[b]{2}{*}{ Effect } & \multirow[b]{2}{*}{$d f$} & \multicolumn{3}{|c|}{ Response Time } & \multicolumn{3}{|l|}{$p$ (early) } & \multicolumn{3}{|c|}{ Error Rate } \\
\hline & & $F$ & $M S E$ & $\eta_{\mathrm{p}}^{2}$ & $F$ & $M S E$ & $\eta_{\mathrm{p}}{ }^{2}$ & $F$ & $M S E$ & $\eta_{\mathrm{p}}{ }^{2}$ \\
\hline Response congruency (C) & 1,31 & $145.00^{*}$ & 260,107 & .82 & $84.84^{*}$ & 1,685 & .73 & $40.32^{*}$ & 112 & .57 \\
\hline Target type $(\mathrm{T})$ & 1,31 & $8.13^{*}$ & 25,752 & .21 & $15.07^{*}$ & 179 & .33 & 0.58 & 41 & .02 \\
\hline Presentation $(\mathrm{P})$ & 3,93 & $54.83^{*}$ & 64,420 & .64 & $33.27^{*}$ & 205 & .52 & 1.32 & 30 & .04 \\
\hline $\mathrm{C} \times \mathrm{T}$ & 1,31 & $65.78^{*}$ & 19,639 & .68 & $32.50^{*}$ & 293 & .51 & 1.05 & 54 & .03 \\
\hline $\mathrm{C} \times \mathrm{P}$ & 3,93 & $5.06^{*}$ & 25,230 & .14 & $42.28^{*}$ & 122 & .58 & 1.97 & 25 & .06 \\
\hline $\mathrm{T} \times \mathrm{P}$ & 3,93 & $7.82^{*}$ & 22,916 & .20 & $11.39^{*}$ & 55 & .27 & 0.30 & 35 & .01 \\
\hline $\mathrm{C} \times \mathrm{T} \times \mathrm{P}$ & 3,93 & 2.41 & 12,283 & .07 & $10.60^{*}$ & 71 & .26 & 1.04 & 19 & .03 \\
\hline
\end{tabular}

${ }^{*} p<.05$ 
than for old congruent targets led to a larger response congruency effect on RT and a higher $p$ (early) value.

\section{General discussion}

The purpose of the present study was to gain a better understanding of the development and characteristics of the nonmediated route for response selection in task-switching situations. In two experiments, subjects switched between origin and size tasks performed on target words. Same-task categories were mapped to different response keys, resulting in targets that were mapped either to the same response (congruent) or to different responses (incongruent) across tasks. Focusing on response congruency, we investigated practice effects at the level of individual target presentations, transfer effects associated with changing category-response mappings, target-specific effects from comparisons of old and new targets during transfer, and the percentages of early responses associated with task-nonspecific response selection as part of advance target processing. In the following sections, we summarize and discuss the results in the context of answering our two questions of interest regarding the nonmediated route.

What is the time course of learning for the nonmediated route?

The training data were similar across experiments (see Figs. 2, $3,4,5,6$ and 7) and indicate that the nonmediated route quickly became functional in response selection. RT rapidly became shorter and $p$ (early) progressively increased for congruent targets with practice, reaching asymptotic levels after 15 presentations. These results suggest faster learning than that observed in a recent study by Forrest, Monsell, and McLaren (2014). In their experiments, for subjects who were instructed to learn and use target-response associations, RTs for congruent targets reached asymptote after about three pairs of blocks, which corresponded to an estimated 93 presentations of each target. For subjects who were instructed about distinct categorization tasks but not explicitly told about the target-response associations, RTs for congruent targets showed more gradual improvement across five pairs of blocks, suggesting an even slower learning rate. It is possible that presenting the target before the cue in our experiments facilitated learning in the nonmediated route, as compared with presenting the cue before the target in Forrest et al.'s experiments. The utility of retrieving target-response instances from memory for selecting responses might be more salient for subjects with target-cue order (in which the target is the sole stimulus available for initial processing) than with cue-target order (in which cue processing might interact with subsequent target processing). Consequently, we think the time course of learning for the nonmediated route depends on when the stimuli are available for processing, the instructions for processing them, and other aspects of the experimental context. We suggest that our experiments may provide one of the fastest estimates of the rate of learning, which can then be compared with the estimates obtained in other experimental contexts to determine how other variables affect the learning process. Thus, we do not contend that the specific time course of learning observed in the present study can be generalized to all task-switching situations that might involve the nonmediated route; instead, we offer our observations as points for comparison with nonmediated learning under other conditions.

Our results suggest that the nonmediated route became faster and contributed more to response selection for congruent targets in our experiments as the number of homogeneous target-response instances in memory increased, consistent with instance-based theories of automaticity (Logan, 1988, 2002) and abstractionist learning theories. The nonmediated route was likely active for incongruent targets, too, but its functionality was limited by the heterogeneous targetresponse instances in memory, which would not improve with practice. That is, learning more instances would not benefit the nonmediated route for incongruent targets because response conflict would persist throughout training. We argue that the performance improvements observed for incongruent targets reflect the increasing efficiency of target encoding and categorization via the mediated route for response selection. The reliable response congruency effect for the first target presentation represents unequivocal evidence that the mediated route was involved in performance (see Schneider, 2015). We think the mediated route continued to play a prominent role in response selection for incongruent targets throughout training, whereas its role diminished for congruent targets as the nonmediated route became functional and faster. Thus, the training results answer our first question by showing the time course over which the nonmediated route is learned, specifically in the context of target-cue order. A more generalizable answer to the question might be provided by comparing target-cue and cue-target orders in task-switching research, which has been a fruitful endeavor both in the distant past (e.g., Bernstein \& Segal, 1968; Biederman, 1973; Davis \& Taylor, 1967; Shaffer, 1965, 1966) and more recently (e.g., Schneider \& Logan, 2014).

Viewed through the lens of the instance theory of automaticity (Logan, 1988, 2002), the mediated and nonmediated routes may operate independently and in parallel, racing against each other to select a response on each trial. Early in training, when there were few target-response instances to retrieve from memory via the nonmediated route, response selection was likely based on categorization via the mediated 
route. As the number of target-response instances racing to be retrieved from memory increased during training, the nonmediated route became progressively faster. ${ }^{2}$ Later in training, when many target-response instances were racing to be retrieved from memory, response selection for congruent targets was likely based mainly on retrieval via the nonmediated route, whereas the mediated route continued to be the basis of response selection for incongruent targets.

Another view of the relationship between the mediated and nonmediated routes is provided by a connectionist model proposed by Ramamoorthy and Verguts (2012). In their model, the mediated route is represented by a pathway in which fast Hebbian learning of task instructions leads to associations between categorical target representations and response representations. The nonmediated route is represented by a pathway that involves slow Hebbian learning of direct associations between targets and responses, based on encoding regularities in the mediated route. Early in training, when target-response associations are weak in the nonmediated route, response selection is based on the mediated route. As specific targets and responses co-occur in the mediated route during training, the target-response associations in the nonmediated route gradually become stronger, presumably to a greater extent for congruent than for incongruent targets. Later in training, the target-response associations are sufficiently strong to enable fast, direct response selection via the nonmediated route. Ramamoorthy and Verguts demonstrated that their model could partially account for response congruency data from Waszak et al. (2008) that implicated the nonmediated route in task-switching performance.

As noted by Schneider and Logan (2014), alternative dualroute models of response selection are also possible. For example, subjects may strategically choose to use one route or the other, with strategy selection modulated by past experience, such that the nonmediated route is chosen more often than the mediated route after training (Rickard, 1997). As another example, the two routes may operate in series, with response selection by the mediated route occurring only if an initial attempt by the nonmediated route fails (Anderson, 2007; Anderson \& Lebiere, 1998). Empirical evidence beyond that provided by the present study will be needed to distinguish between these different types of dual-route models.

\footnotetext{
$\overline{2}$ As described by Logan (1988, p. 495): "The memory process is itself a race. Each stored episode [or instance] races against the others, and the subject can respond on the basis of memory as soon as the first episode is retrieved. The race can be modeled by assuming that each episode has the same distribution of finishing times. Thus, the finishing time for a retrieval process involving $N$ episodes will be the minimum of $N$ samples from the same distribution, which is a well-studied problem in the statistics of extremes."
}

How are responses represented in the nonmediated route?

The transfer data for old targets differed across experiments (see Figs. 2, 3, 4, 5, 6 and 7) in ways that indicate the nature of the response representation in the nonmediated route. In Experiment 1, in which response congruency was maintained from training to transfer, performance impairments were short-lived, and the response congruency effect remained stable for old targets. These results suggest that responses were represented in target-response instances in memory by abstract response labels coding response congruency ("same" or "different" responses across tasks). Evidently, these labels could be rapidly remapped to different manual responses when the category-response mappings changed, resulting in the continued functionality of the nonmediated route and preservation of the response congruency effect. In Experiment 2, in which response congruency switched from training to transfer, performance impairments were greater, and an inverted response congruency effect emerged for old targets (see Wendt \& Kiesel, 2008). These results support the idea that abstract response labels coded response congruency in target-response instances because that information became invalid when the category-response mappings changed in Experiment 2. Evidently, these labels could not be readily rewritten to reflect the switched response congruency information, resulting in impaired functionality of the nonmediated route and inversion of the response congruency effect. Thus, the transfer results answer our second question by suggesting that responses are represented in the nonmediated route by abstract response labels coding response congruency. Given that inverted response congruency effects were observed during transfer with target-cue order in the present study and with cue-target order in Wendt and Kiesel's study, we think our conclusions about the nature of response representations in the nonmediated route may generalize across stimulus orders.

Comparisons between old and new targets during transfer provide additional insight regarding the functioning of the mediated and nonmediated routes. In both experiments, RTs were shorter and $p$ (early) was higher for new targets at the start of transfer than for old targets at the start of training (see Figs. 2, 3, 4, 5, 6 and 7). These results can be attributed to task familiarity and improved efficiency of the mediated route, both of which also contributed to the performance changes seen for incongruent targets during training. By the end of transfer in Experiment 1, which involved only four target presentations, performance for new targets was nearly equivalent to that for old targets. The similar performance for new and old incongruent targets reflects operation of the mediated route. The rapid convergence of performance for new and old congruent targets may reflect a strategic adjustment involving the nonmediated route. That is, metacognitive monitoring of performance during training may have led subjects to realize the benefits of direct 
retrieval via the nonmediated route, leading them to give it more emphasis during transfer. A similar strategy adjustment may have been less effective in Experiment 2, in which the nature of the change in category-response mappings disrupted the nonmediated route for old targets. Old targets that had been congruent during training became incongruent during transfer, forcing a transition from the nonmediated to the mediated route as the principal mode of response selection. Old targets that had been incongruent during training became congruent during transfer, but the heterogeneous set of target-response instances stored in memory during training likely impaired the functionality of the nonmediated route. In contrast, new targets were not subject to any transition or impairment based on prior experience, resulting in performance for new congruent targets that exceeded that for both types of old targets by the end of transfer. Collectively, the results concerning old and new targets shed light on how the mediated and nonmediated routes operate when category-response mappings change after practice.

\section{Conclusion}

The results of the present study provide answers to two important questions about the nonmediated route for response selection in task-switching situations, particularly when advance target processing is possible. First, the training results suggest that the nonmediated route is quickly learned in the context of targetcue order and becomes increasingly involved in response selection with practice, reaching asymptotic performance after a target has been presented 15 times. This rapid rate of learning might represent a useful comparison point for evaluating learning of the nonmediated route in other experimental contexts, such as those involving cue-target order. Second, the transfer results suggest that the target-response instances underlying the nonmediated route involve abstract response labels coding response congruency. When category-response mappings change after practice, the labels can be rapidly remapped to alternative responses if response congruency is maintained (Experiment 1), but the labels cannot be readily rewritten if response congruency is switched (Experiment 2). The results also indicate that subjects can engage in advance target processing to the completion of response selection when they are given the opportunity to do so. Further research addressing the nonmediated route and its relationship with the mediated route may provide additional insight regarding how the cognitive system makes use of different avenues for selecting responses in the context of task switching.

Author note This research was based on part of the first author's doctoral dissertation, supervised by the second author. Funding was provided by the National Institute of Mental Health, through Grant Number R01MH073878-01 to the second author.

\section{Appendixes}

Appendix A

Table 7

Table A1 Target words

\begin{tabular}{lllll}
\hline Set & $\begin{array}{l}\text { Living and } \\
\text { Small }\end{array}$ & $\begin{array}{l}\text { Living and } \\
\text { Large }\end{array}$ & $\begin{array}{l}\text { Nonliving } \\
\text { and Small }\end{array}$ & $\begin{array}{l}\text { Nonliving } \\
\text { and Large }\end{array}$ \\
\hline A & algae & camel & bracelet & bathtub \\
& chickadee & dolphin & dime & boulder \\
& flea & elephant & handcuffs & iceberg \\
frog & leopard & lipstick & patio \\
& ladybug & pony & mug & pier \\
& mouse & shark & peg & sofa \\
& robin & walrus & sock & tuba \\
spider & zebra & wallet & wheelbarrow \\
ant & baboon & bead & asteroid \\
& bacteria & cheetah & cigar & bicycle \\
beetle & donkey & earring & canoe \\
dragonfly & elk & eraser & castle \\
mosquito & otter & mitten & dorm \\
rat & panther & pebble & escalator \\
snail & tiger & scalpel & gym \\
& wasp & wolf & spoon & raft \\
& & & & \\
& & & & \\
& & & &
\end{tabular}

Appendix B

We conducted secondary analyses of the RT data from the training phase to investigate whether the response congruency effect on trial $n$ was modulated by the response congruency on trial $n-1$. Previous research has shown that response congruency effects (and other compatibility effects) on trial $n$ tend to be larger when trial $n-1$ was congruent than when it was incongruent (e.g., Gratton, Coles, \& Donchin, 1992; Kerns et al., 2004; Kiesel, Kunde, \& Hoffmann, 2006; for a review, see Egner, 2007). For these analyses, we excluded the first trial of each successive pair of mini-blocks because it lacked an immediately preceding trial for assessing sequential effects. To obtain an adequate number of observations per condition, we grouped the mini-blocks into five mega-blocks representing Target Presentations 1-4, 5-8, 9-12, 13-16, and 17-20. Mean RTs were submitted to repeated measures ANOVAs with Current Response Congruency (on trial $n$ ), Previous Response Congruency (on trial $n-1$ ), and Mega-Block as factors. For brevity, we report and discuss only the significant effects involving previous response congruency. 


\section{Experiment 1}

RT on trial $n$ was longer when trial $n-1$ was incongruent $(1,464 \mathrm{~ms})$ than when it was congruent $(1,381 \mathrm{~ms})$, resulting in a significant main effect of previous response congruency, $F(1,23)=46.75, M S E=17,792, p<.05, \eta_{\mathrm{p}}{ }^{2}=.67$. The response congruency effect on trial $n$ was larger when trial $n-1$ was congruent $(637 \mathrm{~ms})$ than when it was incongruent (538 ms), consistent with previous findings and resulting in a significant interaction between current and previous response congruency, $F(1,23)=40.91, M S E=7$, $242, p<.05, \eta_{\mathrm{p}}{ }^{2}=.64$. No other effects involving previous response congruency (including potential interactions with mega-block) were significant.

\section{Experiment 2}

RT on trial $n$ was longer when trial $n-1$ was incongruent $(1,357 \mathrm{~ms})$ than when it was congruent $(1,268 \mathrm{~ms})$, resulting in a significant main effect of previous response congruency, $F(1,31)=80.48, M S E=15,622, p<.05, \eta_{\mathrm{p}}{ }^{2}=.72$. The response congruency effect on trial $n$ was larger when trial $n-1$ was congruent (735 ms) than when it was incongruent (633 ms), consistent with previous findings and resulting in a significant interaction between current and previous response congruency, $F(1,31)=44.45, M S E=$ $9,366, p<.05, \eta_{\mathrm{p}}{ }^{2}=.59$. No other effects involving previous response congruency (including potential interactions with mega-block) were significant.

\section{References}

Anderson, J. R. (2007). How can the human mind occur in the physical universe? New York, NY: Oxford University Press.

Anderson, J. R., \& Lebiere, C. (1998). The atomic components of thought. Mahwah, NJ: Erlbaum.

Bernstein, I. H., \& Segal, E. M. (1968). Set and temporal integration. Perception \& Psychophysics, 4, 233-236. doi:10. 3758/BF03206308

Biederman, I. (1973). Mental set and mental arithmetic. Memory \& Cognition, 1, 383-386. doi:10.3758/BF03198124

Brown, J. W., Reynolds, J. R., \& Braver, T. S. (2007). A computational model of fractionated conflict-control mechanisms in taskswitching. Cognitive Psychology, 55, 37-85. doi:10.1016/j. cogpsych.2006.09.005

Buchler, N. G., Hoyer, W. J., \& Cerella, J. (2008). Rules and more rules: The effects of multiple tasks, extensive training, and aging on taskswitching performance. Memory \& Cognition, 36, 735-748.

Cohen-Kdoshay, O., \& Meiran, N. (2007). The representation of instructions in working memory leads to autonomous response activation: Evidence from the first trials in the flanker paradigm. Quarterly Journal of Experimental Psychology, 60, 1140-1154.

Cohen-Kdoshay, O., \& Meiran, N. (2009). The representation of instructions operates like a prepared reflex: Flanker compatibility effects found in first trial following S-R instructions. Experimental Psychology, 56, 128-133. doi:10.1027/1618-3169.56.2.128
Davis, R., \& Taylor, D. H. (1967). Classification on the basis of conditional cues. Quarterly Journal of Experimental Psychology, 19, 30-36.

Egner, T. (2007). Congruency sequence effects and cognitive control. Cognitive, Affective, \& Behavioral Neuroscience, 7, 380-390. doi: 10.3758/CABN.7.4.380

Forrest, C. L. D., Monsell, S., \& McLaren, I. P. L. (2014). Is performance in task-cuing experiments mediated by task set selection or associative compound retrieval? Journal of Experimental Psychology: Learning, Memory, and Cognition, 40, 1002-1024.

Gratton, G., Coles, M. G. H., \& Donchin, E. (1992). Optimizing the use of information: Strategic control of activation of responses. Journal of Experimental Psychology: General, 121, 480-506. doi:10.1037/ 0096-3445.121.4.480

Kantowitz, B. H., \& Sanders, M. S. (1972). Partial advance information and stimulus dimensionality. Journal of Experimental Psychology, 92, 412-418.

Kerns, J. G., Cohen, J. D., MacDonald, A. W., III, Cho, R. Y., Stenger, V. A., \& Carter, C. S. (2004). Anterior cingulate conflict monitoring and adjustments in control. Science, 303, 1023-1026. doi:10.1126/ science. 1089910

Kiesel, A., Kunde, W., \& Hoffmann, J. (2006). Evidence for task-specific resolution of response conflict. Psychonomic Bulletin \& Review, 13, 800-806. doi:10.3758/BF03194000

Kiesel, A., Steinhauser, M., Wendt, M., Falkenstein, M., Jost, K., Philipp, A. M., \& Koch, I. (2010). Control and interference in task switching-A review. Psychological Bulletin, 136, 849-874. doi: $10.1037 / \mathrm{a} 0019842$

Kiesel, A., Wendt, M., \& Peters, A. (2007). Task switching: On the origin of response congruency effects. Psychological Research, $71,117-125$.

Liefooghe, B., De Houwer, J., \& Wenke, D. (2013). Instruction-based response activation depends on task preparation. Psychonomic Bulletin \& Review, 20, 481-487. doi:10.3758/s13423-013-0374-7

Liefooghe, B., Wenke, D., \& De Houwer, J. (2012). Instruction-based task-rule congruency effects. Journal of Experimental Psychology: Learning, Memory, and Cognition, 38, 1325-1335. doi:10.1037/ a0028148

Logan, G. D. (1988). Toward an instance theory of automatization. Psychological Review, 95, 492-527. doi:10.1037/0033295X.95.4.492

Logan, G. D. (2002). An instance theory of attention and memory. Psychological Review, 109, 376-400. doi:10.1037/0033-295X. 109.2.376

Logan, G. D., \& Etherton, J. L. (1994). What is learned during automatization? The role of attention in constructing an instance. Journal of Experimental Psychology: Learning, Memory, and Cognition, 20, 1022-1050. doi:10.1037/0278-7393.20.5.1022

Logan, G. D., \& Schneider, D. W. (2006). Interpreting instructional cues in task switching procedures: The role of mediator retrieval. Journal of Experimental Psychology: Learning, Memory, and Cognition, 32, 347-363. doi:10.1037/0278-7393.32.3.347

Meiran, N. (2014). The task-cuing paradigm: A user's guide. In J. A. Grange \& G. Houghton (Eds.), Task switching and cognitive control (pp. 45-73). New York, NY: Oxford University Press.

Meiran, N., \& Kessler, Y. (2008). The task rule congruency effect in task switching reflects activated long-term memory. Journal of Experimental Psychology: Human Perception and Performance, 34, 137-157. doi:10.1037/0096-1523.34.1.137

Minear, M., \& Shah, P. (2008). Training and transfer effects in task switching. Memory \& Cognition, 36, 1470-1483. doi:10.3758/ MC.336.8.1470

Monsell, S., Sumner, P., \& Waters, H. (2003). Task-set reconfiguration with predictable and unpredictable task switches. Memory \& Cognition, 31, 327-342. 
Ramamoorthy, A., \& Verguts, T. (2012). Word and deed: A computational model of instruction following. Brain Research, 1439, 54-65.

Reisenauer, R., \& Dreisbach, G. (2013). The impact of task rules on distracter processing: Automatic categorization of irrelevant stimuli. Psychological Research, 77, 128-138.

Rickard, T. C. (1997). Bending the power law: A CMPL theory of strategy shifts and the automatization of cognitive skills. Journal of Experimental Psychology: General, 126, 288-311. doi:10.1037/ 0096-3445.126.3.288

Ruge, H., Braver, T., \& Meiran, N. (2009). Attention, intention, and strategy in preparatory control. Neuropsychologia, 47, 1670-1685.

Schneider, D. W. (2014). Modeling graded response congruency effects in task switching. Acta Psychologica, 153, 160-168. doi:10.1016/j. actpsy.2014.10.006

Schneider, D. W. (2015). Isolating a mediated route for response congruency effects in task switching. Journal of Experimental Psychology: Learning, Memory, and Cognition, 41, 235-245. doi:10.1037/ $\mathrm{x} \operatorname{lm} 0000049$

Schneider, D. W., \& Logan, G. D. (2005). Modeling task switching without switching tasks: A short-term priming account of explicitly cued performance. Journal of Experimental Psychology: General, 134, 343-367.

Schneider, D. W., \& Logan, G. D. (2009). Selecting a response in task switching: Testing a model of compound cue retrieval. Journal of Experimental Psychology: Learning, Memory, and Cognition, 35, 122-136. doi:10.1037/a0013744

Schneider, D. W., \& Logan, G. D. (2014). Modelling response selection in task switching: Testing the contingent encoding assumption. Quarterly Journal of Experimental Psychology, 67, 1074-1095. doi:10.1080/17470218.2013.843009
Shaffer, L. H. (1965). Choice reaction with variable S-R mapping. Journal of Experimental Psychology, 70, 284-288. doi:10.1037/ h0022207

Shaffer, L. H. (1966). Some effects of partial advance information on choice reaction with fixed or variable S-R mapping. Journal of Experimental Psychology, 72, 541-545.

Sudevan, P., \& Taylor, D. A. (1987). The cuing and priming of cognitive operations. Journal of Experimental Psychology: Human Perception and Performance, 13, 89-103. doi:10.1037/0096-1523. 13.1.89

Vandierendonck, A., Liefooghe, B., \& Verbruggen, F. (2010). Task switching: Interplay of reconfiguration and interference control. Psychological Bulletin, 136, 601-626. doi:10.1037/a0019791

Waszak, F., Hommel, B., \& Allport, A. (2003). Task-switching and longterm priming: Role of episodic stimulus-task bindings in task-shift costs. Cognitive Psychology, 46, 361-413. doi:10.1016/S0010-0285 (02)00520-0

Waszak, F., Pfister, R., \& Kiesel, A. (2013). Top-down versus bottom-up: When instructions overcome automatic retrieval. Psychological Research, 77, 611-617. doi:10.1007/s00426-012-0459-3

Waszak, F., Wenke, D., \& Brass, M. (2008). Cross-talk of instructed and applied arbitrary visuomotor mappings. Acta Psychologica, 127, 30-35.

Wendt, M., \& Kiesel, A. (2008). The impact of stimulus-specific practice and task instructions on response congruency effects between tasks. Psychological Research, 72, 425-432.

Wenke, D., Gaschler, R., \& Nattkemper, D. (2007). Instruction-induced feature binding. Psychological Research, 71, 92-106. doi:10.1007/ s00426-005-0038-y 